\title{
Development and Validation of a Model to Predict Acute Kidney Injury Following Wasp Stings: A Multicenter Cohort Study
}

\section{Xin Tang}

Sichuan University West China Hospital

Li Lin

Sichuan University West China Hospital

ying Ying Yang

Sichuan University West China Hospital

shuang Rong Huang

Sichuan University West China Hospital

bei Bei Wang

Sichuan University West China Hospital

Ling Zhang ( $\nabla 18702505871 @ 163 . c o m)$

Sichuan University West China Hospital

\section{Ping Fu}

Sichuan University West China Hospital

\section{Yu Wen}

People's Hospital of Zigong City

xian Ji Xu

Renshou People's Hospital

Jian Xiao

Guanyuan Central Hospital

zheng De Chen

People's Hospital of Jianyang City

zhi Zai Zhu

People's Hospital of Meishan City

\section{Hen Xue}

People's Hospital of Ya'an

bin Chang Liao

Third People's Hospital of Zigong City

hong Wei Bi

Third People's Hospital of Mianyang City

\section{Ying Ma}


Zigong City Fourth People's Hospital

\section{Hua Tan}

Sichuan Armed Police Corps Hospital

\section{gang Zhi Tang}

Zigong First People's Hospitall

chao Wan Zhang

Yibin First People's Hospital

qiong De Xie

Yibin Second People's Hospital

\section{Lin Zhang}

Mianyang Central Hospital

\section{feng Lin Liu}

Dujiangyan People's Hospital

\section{ming Fang Chen}

Dazhou Integrative Medicine Hospital

\section{long Yin Wang}

Cangxi People's Hospital

\section{Research}

Keywords: wasp stings, acute kidney injury, risk factors, nomogram, predictive formula

Posted Date: December 14th, 2020

DOI: https://doi.org/10.21203/rs.3.rs-125443/v1

License: (c) (1) This work is licensed under a Creative Commons Attribution 4.0 International License.

Read Full License 


\section{Abstract}

\section{Background}

Acute kidney injury (AKI) following wasp stings is a serious and common health hazard, however the early prediction remains challenging. The study aimed to establish a model to predict AKI following wasp stings and validate it.

\section{Methods}

In the multicenter prospective cohort study, 508 patients with wasp stings from Jul 2015 to Dec 2019 were randomly divided into the training set $(n=381)$ and validation set $(n=127)$ for internal and external validation. A model that based on the multivariable logistic regression analysis was utilized to predict the probability of AKI following wasp stings by a predictive formula and a nomogram. The performances of the model were assessed by using the area under the curve (AUC) and accuracy (ACC) of the receiver operating characteristic curve. The calibration curves were utilized for estimating the consistency between the actual observed outcome and the nomogram predicted AKI probability. Decision curve analysis (DCA) demonstrated the net benefit associated with the use of the nomogram-derived probability for the prediction of AKI following wasp stings.

\section{Results}

Number of stings, hemoglobin $(\mathrm{HB})<110 \mathrm{~g} / \mathrm{dl}$, total bilirubin $(\mathrm{TBI})>34 \mathrm{mg} / \mathrm{dl}$, alanine transaminase $($ ALT $)>40 \mathrm{U} / \mathrm{L}$ and activated partial thromboplastin time (APTT) $>47 \mathrm{~s}$ were demonstrated as the independent risk factors for AKI following wasp stings (all $P$ value $<0.05$ ) and were incorporated into the model. The performances of the model were validated $(A U C=0.912, A C C=0.869$ and $A U C=0.936, A C C=$ 0.898 in the training set and validation set respectively). The predictive formula and nomogram of the model could be utilized to predict the AKI following wasp stings, which having sufficient accuracies, good predictive capabilities and good net benefits.

\section{Conclusion}

In conclusion, we proved that number of stings, $\mathrm{HB}<110 \mathrm{~g} / \mathrm{dl}, \mathrm{TBI}>34 \mathrm{mg} / \mathrm{dl}, \mathrm{ALT}>40 \mathrm{U} / \mathrm{L}$ and APTT > $47 \mathrm{~s}$ were independence risk factors for AKI following wasp stings. The predictive formula and the individual nomogram of the model might serve as promising predictive tools to assess the probability of the AKI following wasp stings.

\section{Introduction}

Wasps and bees are the members of the order Hymenoptera, suborder Apocrita, and superfamily Vespoidea[1], and the wasp or bee stings are frequently reported [2-6]. Wasp or bee stings may cause acute kidney injury (AKI), intravascular hemolysis, rhabdomyolysis and even death[7-10]. The incidence rate of AKI following wasp stings ranges from $20-50 \%[11-13]$. According to a study reported, of patients 
who with AKI following wasp stings, $20 \%-58 \%$ patients are developed to chronic kidney disease (CKD)[12, 14] and 5.6-50.5\% patients die although some of them receiving renal replacement therapy (RRT)[1315].

At present, the mechanism of AKI following wasp stings is not completely clear. Wasp venoms is thought having direct toxic effect for kidney, in which main effective poisonous components include phospholipase $A_{2}\left(P L A_{2}\right)$ and melittin $[11,16,17]$. Besides, hemoglobin $(H B)$ released by hemolysis and myoglobin released by rhabdomyolysis are considered that may cause secondary damage to kidney[18, 19].

At present, the AKI following wasp stings is a serious health hazard[17]. Besides, it's worth noting that our previous study indicated that patients with AKI following wasp stings usually died within 72 hours after admission, so that a better understanding of AKI following wasp stings is helpful to early diagnose and treat[12]. Therefore, we performed a multicenter center prospective cohort to identify risk factors associated with AKI following wasp stings and established an individual nomogram and formula of the model to predict the probability of AKI following multiple wasp stings.

\section{Materials And Methods}

\section{Database and Patient Selection}

This was a multicenter prospective observational cohort study that was conducted in 18 hospitals in China from Jul 2015 to Dec 2019 and 508 patients with AKI following wasp stings were included. Patients who suffering from stings by wasp or other bee species, whether AKI occurred or not, were included. Patients who met the following criteria were excluded: (1) lack of enough clinical data to analyze; or (2) disapprove of participating. The study was approved by Institutional Research Ethics Committees of our institution (Approval No. of the ethics committee: 2014-156). Written informed consents were obtained from all included patients or their legally authorized representatives.

The data were recorded and collected from the electronic medical records system. Demographic and clinical characteristics, such as age, sex, number of stings, time from sting to admission, Sequential Organ Failure Assessment (SOFA) score et al, and important laboratory measures, such as the lactated dehydrogenase (LDH), HB, total bilirubin (TBI), aspartate aminotransferase (AST), urine volume, alanine aminotransferase (ALT), and activated partial thromboplastin time (APTT), creatine kinase (CK) and serum creatinine (Scr) et al, were recorded and analyzed.

\section{Definition}

In the study, the patients with wasp stings were divided into AKI group or non-AKI group. AKI was defined by the 2012 Kidney Disease Improving Global Outcomes guidelines[20]: (1) Scr increased to $\geq 26.5 \mu \mathrm{mol} /$ $\mathrm{L}(0.3 \mathrm{mg} / \mathrm{dL})$ within 48 hours; (2) Scr increased to 1.5 times within 7 days ; or (3) urine volume $<0.5 \mathrm{ml} /$ $(\mathrm{kg} \cdot \mathrm{h})$ for 6 hours. 
In the logistic regression analysis, continuous variables were transformed into categorical variables according to their reference range and common clinical transformation methods.

\section{Statistical Analysis}

We excluded some variables that missing more than $15 \%$ and interpolated some variables that missing less than $15 \%$ according to multiple interpolation method. And some patients were excluded for lack of enough data to analyze. All eligible patients were randomly assigned 3:1 into the training set and the validation set. The training set mainly constructed a nomogram and a predictive formula of the model for predicting the AKI following multiple wasp stings and the validation set was constructed for external validation.

Continuous variables are expressed as the mean (standard deviation [SD]) which were compared by t test or the median (interquartile range [IQR]) which were compared by Wilcoxon test according to whether the variable coincided with a normal distribution. Categorical variables were expressed as proportions, which were compared by the chi-squared test or Fisher's exact test as appropriate.

The univariable logistic regression analysis to detect the risk factors of AKI following wasp stings and the variables with $\mathrm{P}$-value $<0.1$ were introduced into the multivariable logistic regression analysis. In addition, the individual nomogram and the predictive formula of the model were constructed according to the result of multivariable logistic regression analysis. The predictive formula was constructed according to the previous study[21]. The internal validation and external validation were performed in the training set

and validation set respectively to assess the accuracy of the model by a bootstrap validation method with 200 resamples. The receiver operating characteristic curve (ROC) analysis was performed to assess the model. Cut off of the model was obtained based on Youden Index. The calibration curves were utilized for estimating the consistency between the actual observed outcome and the nomogram predicted AKI probability. Decision curve analysis (DCA) demonstrated the net benefit associated with the use of the nomogram-derived probability for the prediction of AKI following wasp stings.

All statistical analyses were performed by SPSS (SPSS Inc., Chicago, IL) software for Windows version 23.0. and the packages (rms, hmisc, etc.) in R software version 3.6.1 (http://www.r-project.org), with a two-sided $P$ value $<0.05$ considered statistically significant.

\section{Results}

\section{Characteristics of Eligible Patients}

In the study, 547 patients with wasp stings were screened, and 39 patients were excluded due to missing necessary dada (as shown in the Fig. 1). Finally, 508 eligible patients were randomly assigned into the training set $(n=381)$ and the validation set $(n=127)$. There was no significant difference between the training set and the validation set in the distributions of demographic and disease characteristics such as year, gender, number of AKI, condition of wasp stings (see Table A1 in Additional file 1). In the training set, 
of the 381 patients, 118 (31.0\%) patients with AKI and 263 (69.0\%) without AKI. The demographic and clinical characteristics between the AKI group and non-AKI group in the training set were presented in the Table 1. The proportion of patients received renal replacement therapy (RRT) were higher and the time from stings to admission was longer in the AKI group than those in the non-AKI group (all $P$ value $<0.05$ ). Meanwhile, the proportion of wasp stings, sting at head and face, number of stings $\geq 30$, area of stings $\geq 25 \%$ and the level of ALT, AST, LDH, TBI, CK in the AKI group were higher than those in the non-AKI group (all $P$ value $<0.05$ ). 
Table 1

Demographic and disease characteristics of patient in training set

\begin{tabular}{|c|c|c|c|c|}
\hline Variables & Overall (381) & No AKI (263) & AKI (118) & $P$-value \\
\hline Year, y & $55.2(15.4)$ & $54.2(16.5)$ & $57.3(12.7)$ & 0.07 \\
\hline Gender, No (\%) & & & & 0.06 \\
\hline Male & $191(50.1)$ & $123(46.8)$ & $68(57.6)$ & \\
\hline Female & 190 (49.9) & $140(53.2)$ & $50(42.4)$ & \\
\hline RRT, No. (\%) & & & & $<0.001$ \\
\hline No & $229(60.1)$ & $207(78.7)$ & $22(18.6)$ & \\
\hline Yes & $152(39.9)$ & $56(21.3)$ & $96(81.4)$ & \\
\hline Time from stings to admission, $\mathrm{h}$ & $14.0(33.0)$ & $9.8(28.7)$ & $23.5(39.6)$ & $<0.001$ \\
\hline Wasp, No (\%) & & & & 0.04 \\
\hline No & $141(37.0)$ & $107(40.7)$ & $34(28.8)$ & \\
\hline Yes & $240(63.0)$ & $156(59.3)$ & $84(71.2)$ & \\
\hline Sting at head and face & & & & 0.001 \\
\hline No & $71(18.6)$ & $61(23.2)$ & $10(8.5)$ & \\
\hline Yes & $310(81.4)$ & $202(76.8)$ & $108(91.5)$ & \\
\hline Number of stings, No (\%) & & & & $<0.001$ \\
\hline$\leq 100$ & $36(9.4)$ & $10(3.8)$ & $26(22.0)$ & \\
\hline$\leq 50$ & $38(10.0)$ & $11(4.2)$ & $27(22.9)$ & \\
\hline$\leq 30$ & $72(18.9)$ & $36(13.7)$ & $36(30.5)$ & \\
\hline$\leq 15$ & 235 (61.7) & $206(78.3)$ & $29(24.6)$ & \\
\hline Area of stings, No (\%) & & & & $<0.001$ \\
\hline$\leq 100 \%$ & $15(3.9)$ & $6(2.3)$ & $9(7.6)$ & \\
\hline$\leq 50 \%$ & $48(12.6)$ & $13(4.9)$ & $35(29.7)$ & \\
\hline$\leq 25 \%$ & 79 (20.7) & $43(16.3)$ & $36(30.5)$ & \\
\hline$\leq 10 \%$ & $239(62.7)$ & $201(76.4)$ & $38(32.2)$ & \\
\hline SBP, mmHg & $144.3(56.1)$ & $138.1(24.2)$ & $157.9(92.9)$ & 0.001 \\
\hline $\mathrm{DBP}, \mathrm{mmHg}$ & $86.1(15.5)$ & $84.1(15.4)$ & $90.6(14.9)$ & $<0.001$ \\
\hline Creatinine, mg/dl & 70 (46.3) & $65.8(24.2)$ & 180.5(214) & $<0.001$ \\
\hline
\end{tabular}




\begin{tabular}{|c|c|c|c|c|}
\hline Variables & Overall (381) & No AKI (263) & AKI (118) & $P$-value \\
\hline Urine volume, $\mathrm{ml} / 24 \mathrm{~h}$ & 1461.5 (826.9) & $1757.2(501.2)$ & $802.2(1011.5)$ & $<0.001$ \\
\hline Hemoglobin, g/dl & $132.8(36.7)$ & 135.8 (19.4) & $126.2(58.9)$ & 0.02 \\
\hline Leukocyte, X10\%/L & $16.8(17.3)$ & $13.0(8.6)$ & $25.3(26.5)$ & $<0.001$ \\
\hline Platelet, $X 10^{9} / \mathrm{L}$ & $175.0(71.5)$ & $170.6(64.3)$ & $184.8(84.8)$ & 0.07 \\
\hline TBI, mg/dl & $22.8(33.7)$ & $18.9(13.1)$ & $66.6(55.7)$ & $<0.001$ \\
\hline $\mathrm{ALT}, \mathrm{U} / \mathrm{L}$ & $36.1(65.1)$ & $26(34.2)$ & 154.5(321.5) & $<0.001$ \\
\hline AST, U/L & $52.4(213.0)$ & $32.6(50.8)$ & 686.5(1121.4) & $<0.001$ \\
\hline$T P, g / L$ & $68.2(11.3)$ & $67.7(7.7)$ & $69.3(16.7)$ & 0.18 \\
\hline Albumin, g/L & $42.1(5.0)$ & $42.7(4.3)$ & $40.5(5.9)$ & $<0.001$ \\
\hline $\mathrm{UA}, \mu \mathrm{mol} / \mathrm{L}$ & 337.8 (121.5) & 312.2 (89.4) & 394.8 (159.2) & $<0.001$ \\
\hline $\mathrm{CK}, \mathrm{U} / \mathrm{L}$ & 317 (1047.2) & $195(562.8)$ & 2656.5(5274.5) & $<0.001$ \\
\hline $\mathrm{LDH}, \mathrm{U} / \mathrm{L}$ & 309 (1045.2) & $235.1(143.0)$ & 2051.5(1781.3) & $<0.001$ \\
\hline Potassium, mmol/L & $4.1(1.9)$ & $3.9(2.2)$ & $4.5(0.9)$ & 0.008 \\
\hline Calcium, mmol/L & $2.25(0.17)$ & $2.28(0.14)$ & $2.18(0.19)$ & $<0.001$ \\
\hline PT, s & $14.3(9.9)$ & $14.3(11.0)$ & $14.4(6.7)$ & 0.9 \\
\hline APTT, s & $51.1(28.6)$ & $44.1(21.2)$ & $66.8(36.0)$ & $<0.001$ \\
\hline \multicolumn{5}{|c|}{$\begin{array}{l}\text { Data represent the mean (SD) or median (interquartile ranges) as appropriate; categorical data } \\
\text { represent percentage }\end{array}$} \\
\hline \multicolumn{5}{|l|}{ AKI: acute kidney injury } \\
\hline \multicolumn{5}{|c|}{ RRT: renal replacement therapy } \\
\hline \multicolumn{5}{|l|}{ SBP: systolic pressure } \\
\hline \multicolumn{5}{|c|}{ DBP: diastolic blood pressure } \\
\hline \multicolumn{5}{|l|}{ TBI: total bilirubin } \\
\hline \multicolumn{5}{|c|}{ ALT: alanine transaminase } \\
\hline \multicolumn{5}{|c|}{ AST: aspartate aminotransferase } \\
\hline \multicolumn{5}{|l|}{ TP: total protein } \\
\hline \multicolumn{5}{|l|}{ UA: uric acid } \\
\hline CK: creatine kinase & & & & \\
\hline
\end{tabular}


LDH: lactate dehydrogenase

PT: prothrombin time

APTT: activated partial thromboplastin time

\section{Risk Factors Related to AKI following wasp stings}

To determine the risk factors for AKI following wasp stings, the univariable and multivariable logistic regression analysis were performed in the training set. Variables with $P<0.1$ in the univariable logistic regression analysis introduced into multivariable logistic regression analysis (see Table A2 in Additional file 2). Subsequently, multivariable logistic regression analysis identified five variables as independence risk factors for AKI following wasp stings: number of stings, $\mathrm{HB}<110 \mathrm{~g} / \mathrm{dl}, \mathrm{TBI}>34 \mathrm{mg} / \mathrm{dl}, \mathrm{ALT}>40 \mathrm{U} / \mathrm{L}$ and APTT > $47 \mathrm{~s}$ (as shown in the Table 2).

Table 2

Multivariable logistic regression analysis results to predict AKI following wasp stings

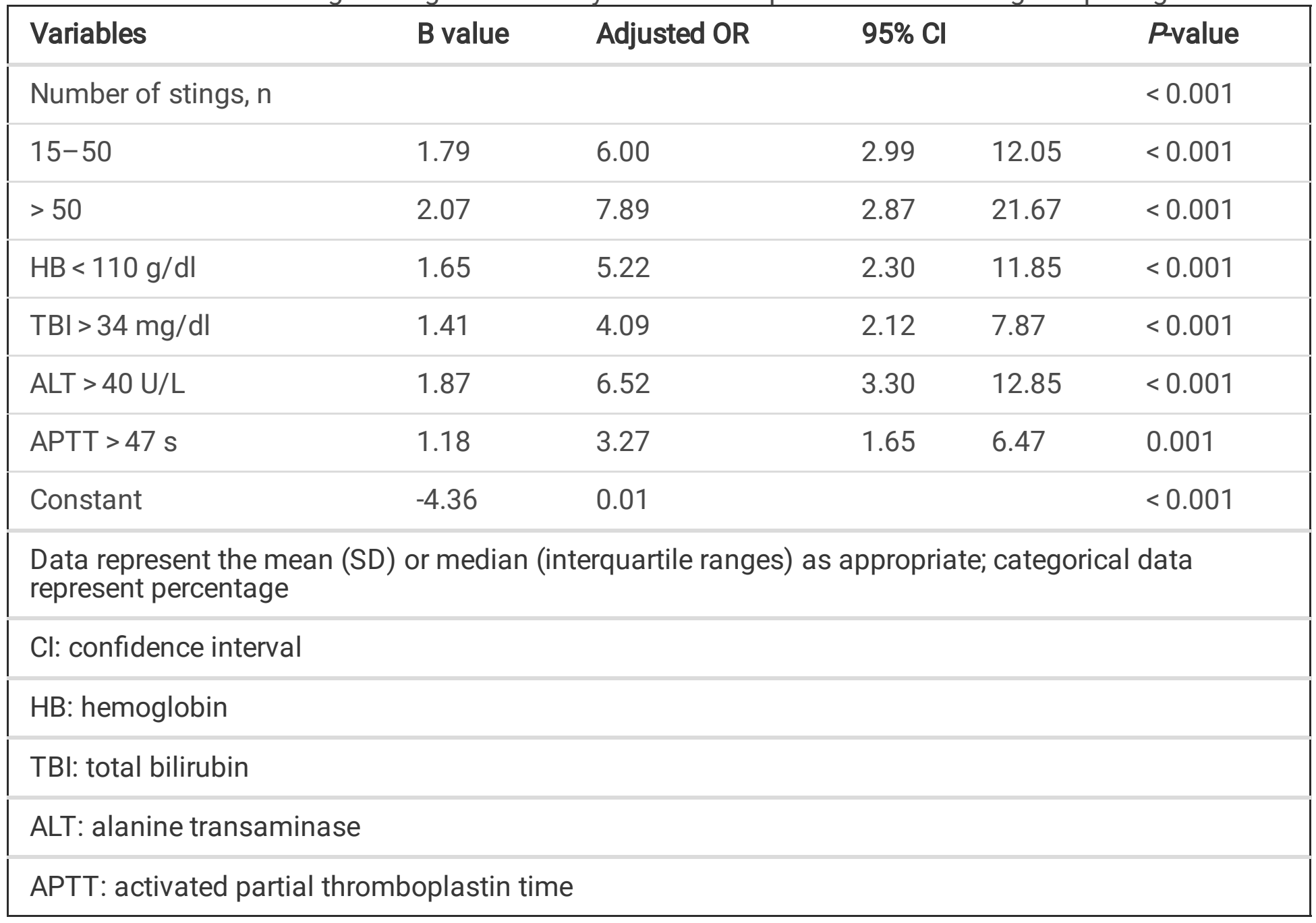




\section{Construction of a predictive formula according to multivariable logistic regression analysis}

According to multivariable logistic regression, we could construct the predictive formula of the model to predict the AKI for multiple wasp sting patients in the training set:

$P$ (for AKI following wasp stigns)

$$
=\frac{1}{1+e^{4.36-1.79 X 1-2.07 X 2-1.65 \times 3-1.41 X 4-1.87 X 5-1.18 X 6}}
$$

In the formula, $\mathrm{P}$ indicated the predicted $\mathrm{AKI}$ probability, $\mathrm{X}$ indicated the variables that included in the model (X1-X6 represented that number of stings is $15-50$, number of stings $>50, \mathrm{HB}<110 \mathrm{~g} / \mathrm{dl}, \mathrm{TBI}>$ $34 \mathrm{mg} / \mathrm{dl}, \mathrm{ALT}>40 \mathrm{U} / \mathrm{L}, \mathrm{APTT}>47 \mathrm{~s}$ respectively). $\mathrm{X}$ was assigned as 1 while patient was consistent with the variable, otherwise, $X$ was assigned as 0 . The probability of AKI following multiple wasp stings could be calculated according to the formula based on the patient's individual clinical characteristics. When calculated probability $>0.338$ (the cut off of the model), the AKI was predicted to occur, and when calculated probability $\leq 0.338$, the AKI might not occur.

Of note, as shown in the Table 3, the model showed a higher predictive value and diagnostic accuracy (area under the curve $[A U C]=0.912$, accuracy $[A C C]=0.869$ ) than any other risk factors such as ALT (AUC $=0.745, \mathrm{ACC}=0.772), \mathrm{APTT}(\mathrm{AUT}=0.708, \mathrm{ACC}=0.703), \mathrm{HB}(\mathrm{AUC}=0.594, \mathrm{ACC}=0.717)$, number of stings $($ AUC $=0.778, A C C=0.774)$ and TBI $($ AUC $=0.777, A C C=0.795)($ all $P$ value $<0.05)$. The cut off of the model was 0.338 . In addition, the specificity of the model was 0.882 , the sensitivity of the model was 0.856 and the prediction accuracy was 0.869 . The similar results were found in the validation set (as shown in the Table 3 and Figure A1 in Additional file 3). 
Table 3

Performance of prediction model and independence risk factors

\begin{tabular}{|c|c|c|c|c|c|c|c|}
\hline Variables & AUC & \multicolumn{2}{|c|}{$95 \% \mathrm{Cl}$} & Specificity & Sensitivity & ACC & $P$-value ${ }^{a}$ \\
\hline \multicolumn{8}{|c|}{ Prediction model } \\
\hline Training set & 0.912 & 0.88 & 0.94 & 0.882 & 0.856 & 0.869 & \\
\hline Validation se & 0.936 & 0.88 & 0.97 & 0.922 & 0.865 & 0.898 & \\
\hline \multicolumn{8}{|l|}{ ALT } \\
\hline Training set & 0.745 & 0.70 & 0.79 & 0.684 & 0.805 & 0.772 & $<0.001$ \\
\hline Validation se & 0.755 & 0.67 & 0.83 & 0.644 & 0.865 & 0.709 & $<0.001$ \\
\hline \multicolumn{8}{|l|}{ APTT } \\
\hline Training set & 0.708 & 0.66 & 0.75 & 0.696 & 0.720 & 0.703 & $<0.001$ \\
\hline Validation se & 0.705 & 0.62 & 0.78 & 0.733 & 0.676 & 0.717 & $<0.001$ \\
\hline \multicolumn{8}{|l|}{$\mathrm{HB}$} \\
\hline Training set & 0.594 & 0.54 & 0.64 & 0.916 & 0.271 & 0.717 & $<0.001$ \\
\hline Validation se & 0.532 & 0.44 & 0.62 & 0.956 & 0.108 & 0.709 & $<0.001$ \\
\hline \multicolumn{8}{|c|}{ Number of stings } \\
\hline Training set & 0.778 & 0.73 & 0.82 & 0.783 & 0.754 & 0.774 & $<0.001$ \\
\hline Validation se & 0.836 & 0.76 & 0.90 & 0.822 & 0.811 & 0.819 & 0.005 \\
\hline \multicolumn{8}{|l|}{ TBI } \\
\hline Training set & 0.777 & 0.73 & 0.82 & 0.825 & 0.729 & 0.795 & $<0.001$ \\
\hline Validation se & 0.885 & 0.82 & 0.94 & 0.878 & 0.892 & 0.882 & 0.01 \\
\hline
\end{tabular}

a: The P value was obtained by compared the AUC between the prediction model and ALT, APTT, HB, Number of stings or TBI respectively.

AUC: area under the curve

Cl: confidence interval

ACC: accuracy

ALT: alanine transaminase

APTT: activated partial thromboplastin time

HB: hemoglobin

TBI: total bilirubin 


\section{Construction of a Nomogram and Validation}

The individual nomogram was constructed based on the result of the multivariable logistic regression analysis to predict AKI for wasp sting patients in the training set too. As shown in the Fig. 2 and Table A3 in Additional file 4, scores were signed for each variable. A total point could be calculated by adding all points based on patient's individual clinical characteristics, which was lower meaning a lower probability of AKI. The nomogram was validated internally in the training set and were validated externally in the validation set. The calibration curves for AKI prediction showed excellently accordance between predictions of the nomogram and the actual observations in the training set (as shown in the Fig. 3) and validation set (see Figure A2 in Additional file 5). DCA verified the net benefit associated with the use of the nomogram-derived probability for AKI following wasp stings in the training set (as shown in the Fig. 3) and validation set (see Figure A3 in Additional file 6).

\section{Discussion}

In our study, number of stings, $\mathrm{HB}<110 \mathrm{~g} / \mathrm{dl}, \mathrm{TBI}>34 \mathrm{mg} / \mathrm{dl}, \mathrm{ALT}>40 \mathrm{U} / \mathrm{L}$ and APTT $>47$ s were identified as independent risk factors for AKI following wasp stings according to univariable and multivariable logistic regression analysis in the training set. And the predictive formula and the individual nomogram that included those independent risk factors was developed and validated to predict the probability of the AKI following wasp stings. Those were demonstrated having the sufficient accuracy and good predictive capability based on the internal validation and external validation in the training set and validation set respectively. In addition, the predictive formula and individual nomogram both have clinical significance to assess the probability of the AKI following wasp stings and make a decision for therapy by those easy, convenient and effective methods.

At present, wasp stings are reported frequently, especially in rural areas where patients may have low incomes[22-24], which is a common challenge to society. In the present study, the incidence rate of AKI is $30.5 \%(155 / 508)$ in patients with wasp stings, which is nearly equal to results in the previous studies that reported the incidence rate of AKI is $20-25 \%[11,25,26]$. In addition, the mortality in patients with wasp stings is $5.7 \%(29 / 508)$, however the mortality $(17.4 \%$ [27/155]) in the AKI group is apparently higher than that $(0.6 \%$ [2/353]) in the non-AKI group ( $P<0.001$ as shown Figure A4 in Additional file 7$)$. Those indicated that it is significant to understand, risk predict and early diagnose the AKI following wasp stings. Of note, $55.6 \%(15 / 27)$ patients with AKI following wasp stings died within 72 hours after admission, that is similar to the result of previous study[14]. At present, AKI following wasp stings with relatively high mortality rate and rapid onset were called the "Silent Killer" because it threated to human public health. Therefore, early detection and diagnosis should be performed promptly to help clinicians make therapeutic decisions for obtaining a good prognosis.

However, the prediction model of AKI following wasp stings is rarely reported in previous studies. We established the predictive formula and the individual nomogram based on the independent risk factor, 
which predicted the AKI of patients undergoing wasp stings with a good validation. As reported in previous studies, nomogram is extensively used to predict the probability of a disease or a clinical outcome based on multiple variables[27-30]. In the present study, the visual nomogram could calculated the specific probability of AKI following wasp stings based on the sum of the scores of each risk factor, that is the most user-friendly tool to judge the specific situation of each patient[31]. The nomogram is intuitive and easy-to-understand not only clinicians but for patients as well, which might make it easy for communication between clinicians and patients. Of note, nomograms have never ever been reported for AKI following wasp stings to our knowledge, we conduct the first nomogram to predict the AKI following wasp stings.

Besides, the predictive formula also is conducted to assess whether occur AKI or not in patients with wasp stings, that is judged according to whether the calculated probability > the cut off of the model (0.338) or not. One of the two methods could be selected according to the habits and preferences of clinicians, or both to mutually detect and support the results. Those are composed of common clinical parameters, that are easy to obtain from laboratorial blood tests. In addition, the sufficient accuracy and good predictive capability of the model are verified by AUC of ROC ( 0.912 in the training set and 0.936 in the validation set), and the net benefit is verified by DCA. Therefore, the model might provide a clinical assistance in early recognition, detection, diagnosis and intervention of AKI following wasp stings.

According to previous studies, AKI is induced in wasp stings based on direct toxicity of the venom components, hypotension, intravascular hemolysis and rhabdomyolysis[32]. The venom components, such as PLA $\mathrm{A}_{2}$ that is mainly included in wasp venom and melittin that is the mainly included in the bee venom, both have strong hemolytic toxicity and direct toxic effect for inducing the apoptosis of renal tubule epithelial cells[16,33]. Hypotension might lead to ischemic renal lesion, which is induced by main components of bee venom such as hyaluronidase, apamin and substances induced by those venom themselves such as histamine, serotonin, bradykinin.

Besides, rhabdomyolysis and hemolysis induced AKI by renal vasoconstriction, formation of intratubular deposits of myoglobin and direct cytotoxicity of myoglobin and HB that are release from muscle and red blood cells. However, there is no full understanding in the mechanism through which renal damage occurs. Nonetheless, it is certain that rhabdomyolysis and hemolysis are thought play important roles in AKI following wasp stings.

In the present study, compared with non-AKI group, we actually find the levels of CK, ALT, AST elevate in the AKI group, which might be associated with rhabdomyolysis. We also find LDH increase and anemia ( $\mathrm{HB}$ in the red blood cells decrease) in the AKI group, which might be is concerned with hemolysis. They also are the risk factors of AKI following wasp sting according to univariable logistic regression analysis. Continuous variables are transformed into categorical variables according to their reference range, which is beneficial to full use the results through constructing the model. 
According to multivariable logistic regression analysis, number of stings, $\mathrm{HB}<110 \mathrm{~g} / \mathrm{dl}, \mathrm{TBI}>34 \mathrm{mg} / \mathrm{dl}$, $\mathrm{ALT}>40 \mathrm{U} / \mathrm{L}$ and APTT $>47 \mathrm{~s}$ are considered as independence risk factors and could help to predict AKI in patients with wasp stings. Rhabdomyolysis and hemolysis could induce the level of indirect bilirubin to elevate, which could be one explanation of the increasing of TBI in AKI following wasp stings. According to the study reported, disseminated intravascular coagulation (DIC) might be a possible factor that contributes to AKI, described in rhabdomyolysis. While DIC occurred, thromboplastin is released and micro thrombi is formed in the glomeruli, which causing the consequent glomerular filtration rate reduction[34]. DIC might induce APTT prolonged by increasing the consumption of coagulation factors. We think those might explain why prolonged APTT is an independence risk factors in AKI following wasp stings. In addition, we also find that wasp compared with other bee species, sting at head and face compared with other locations, the greater number of stings and large area of sting might be associated with AKI following wasp stings.

However, a recent study with a retrospective cohort study involving 112 patients conducted by Hai Yuan et al, which showed that elevated leukocytes, high myoglobin, high urinary monocyte chemotactic protein1 (MCP-1) are the independence risk factors of AKI induced by multiple wasp stings[25]. In fact, our results also find that, while compared with non-AKI, the level of leukocytes is higher in the patients with AKI following wasp stings. However, we do not think elevated leukocytes is an independence risk factor of AKI following wasp stings based on the multivariable logistic regression analysis. There exists a difference between the two studies. It's worth noting that the results are obtained based on the single center as well as the small sample size in the Hai Yuan's study. In addition, 12 variables included in the multivariable logistic regression analysis to find out the independence risk factors based on only 54 patients who occurred AKI. We think those might restrict external validity and cause the different results in the Hai Yuan's study. Besides, Hai Yuan's study only reported some independence risk factors, which did not construct a model to predict AKI for fully utilization the result. We construct the first model based on a large data from the multicenter prospective cohort study and validate it.

There exist some limitations in the present study. First, there are some variables missing too much so that we have to exclude them such as cystatin $-\mathrm{C}$ and urine protein, although we already try our best to collect the complete data of each patient. Second, although data were collected from multicenter, the enrolled patients all were of the same ethnicity, which might limit the scalability of the model. Third, we select the variable by forward stepwise in multivariable logistic regression analysis, that might induce the final model that contain terms of little values. Besides, the validation was performed by bootstrapping technology, however there also need further external validation in further.

\section{Conclusion}

In conclusion, we proved that number of stings, $\mathrm{HB}<110 \mathrm{~g} / \mathrm{dl}, \mathrm{TBI}>34 \mathrm{mg} / \mathrm{dl}, \mathrm{ALT}>40 \mathrm{U} / \mathrm{L}$ and APTT > $47 \mathrm{~s}$ were independence risk factors for AKI following multiple wasp stings. And the predictive formula and the individual nomogram were established and validated based on those predictive factors to predict AKI following wasp stings. Of note, those could serve as promising predictive tools to assess the 
probability of the AKI following wasp stings and help make a decision for therapy easily and conveniently.

\section{Declarations}

\section{Ethics approval and consent to participate}

The study was approved by Institutional Research Ethics Committees of West China Hospital of Sichuan University (Approval No. of the ethics committee: 2014-156).

All enrolled patients approved to participant and written informed consents were obtained from all enrolled patients or their legally authorized representatives.

\section{Consent for publication}

All authors approved of publication.

\section{Availability of data and materials}

The datasets used and/or analyzed during the current study are available from the corresponding author on reasonable request

\section{Competing interests}

The authors declare that they have no competing interests

\section{Funding}

1.3.5 project for disciplines of excellence-Clinical Research Incubation Project, West China Hospital, Sichuan University (18HXFH018 and ZYGD18027). Those funded in the design of the study and collection, analysis, and interpretation of data and in writing the manuscript.

\section{Authors' contributions}

All authors contributed to the study conception and design. PF and LZ, as the corresponding authors of this paper, were mainly responsible for program design and modification. XT and LL wrote the first draft. $X T, L L$ and $Y Y$ summarized data from each center. $L Z, B W$ and $R H$ assessed the data and analyzed. AndYW, JXu, JXi, DC, ZZ, HX, CL7, WB, YM, HT, ZT, WZ, DX, LinZ, LL, FC, YW all put their hands-on enrolling patients, recording data and completing patient follow-up. All authors commented on previous versions of the manuscript and all authors read and approved the final manuscript.

\section{Acknowledgements}

Thanks are due to Renshou people's Hospital, Guangyuan Central Hospital, People's Hospital of Jianyang City, People's Hospital of Meishan city, People's Hospital of Ya'an, Third People's Hospital of Zigong City, 
Third People's Hospital of Mianyang city, Zigong City Fourth People's Hospital, Yibin First People's Hospital, Sichuan Armed Police Corps Hospital, Zigong First People's Hospital, Yibin Second People's Hospital, Mianyang Central Hospital, Dujiangyan People's Hospital, Dazhou integrative medicine hospital, Cangxi People's Hospital for participating the study, enrolling patients and collecting data.

\section{References}

1. Zirngibl G, Burrows HL: Hymenoptera stings. Pediatr Rev 2012, 33(11):534-535; discussion 535.

2. Witharana EW, Wijesinghe SK, Pradeepa KS, Karunaratne WA, Jayasinghe S: Bee and wasp stings in Deniyaya; a series of 322 cases. Ceylon Med J 2015, 60(1):5-9.

3. Xie C, Xu S, Ding F, Xie M, Lv J, Yao J, Pan D, Sun Q, Liu C, Chen T et al: Clinical features of severe wasp sting patients with dominantly toxic reaction: analysis of 1091 cases. PLOS One 2013, 8(12):e83164.

4. Kumar R, Kumar C, Khosla $\mathrm{H}$ : An interesting case report of acute renal failure due to wasp bite. $J$ Family Med Prim Care 2019, 8(4):1488-1490.

5. Ryakitimbo A, Kennedy M, Shao E, Itana ME, Mbwasi R, Kinabo G, Yeates K, Kilonzo K: Acute kidney injury in a Tanzanian boy following multiple bee stings in resource-limited setting: a case report. $O x f$ Med Case Reports 2018, 2018(10):omy070.

6. Vikrant S, Parashar A: Two Cases of Acute Kidney Injury Due to Multiple Wasp Stings. Wilderness Environ Med 2017, 28(3):249-252.

7. Vikrant S, Parashar A: Acute kidney injury due to multiple Hymenoptera stings-a clinicopathological study. Clin Kidney J 2017, 10(4):532-538.

8. Sharma M, Das HJ, Barman AK, Mahanta PJ: Acute kidney injury due to multiple wasp stings. Saudi J Kidney Dis Transp/ 2017, 28(1):196-198.

9. Nair BT, Sanjeev RK, Saurabh K: Acute kidney injury following multiple bee stings. Ann Afr Med 2016, 15(1):41-42.

10. Wenjie W, Houqing L, Gengyun S: Rhabdomyolys is owing to a wasp sting. Clin Exp Pharmacol Physiol 2015, 42(7):808-809.

11. Gong J, Yuan H, Gao Z, Hu F: Wasp venom and acute kidney injury: The mechanisms and therapeutic role of renal replacement therapy. Toxicon 2019, 163:1-7.

12. Mejía Vélez G: [Acute renal failure due to multiple stings by Africanized bees. Report on $\mathbf{4 3}$ cases]. \#N/A 2010, 30(5):531-538.

13. Paudel B, Paudel K: A study of wasp bites in a tertiary hospital of western Nepal. Nepal Med Coll J 2009, 11(1):52-56.

14. Zhang $L$, Yang Y, Tang Y, Zhao Y, Cao Y, Su B, Fu P: Recovery from AKI following multiple wasp stings: a case s-eries. Clin J Am Soc Nephrol 2013, 8(11):1850-1856.

15. Bhatta N, Singh R, Sharma S, Sinnha A, Raja S: Acute renal failure following multiple wasp stings. Pediatr Nephrol 2005, 20(12):1809-1810. 
16. Shu Y, Yang Y, Zhao Y, Ma L, Fu P, Wei T, Zhang L: Melittin Inducing the Apoptosis of Renal Tubule Epithelial Cells through Upregulation of Bax/Bcl-2 Expression and Activation of TNF-a Signaling Pathway. Biomed Res Int 2019, 2019:9450368.

17. Silva GBDJ, Vasconcelos AGJ, Rocha AMT, Vasconcelos VR, Barros JN, Fujishima JS, Ferreira NB, Barros EJG, Daher EF: Acute kidney injury complicating bee stings - a review. \#N/A 2017, 59:e25.

18. Dvanajscak Z, Walker PD, Cossey LN, Messias NC, Boils CL, Kuperman MB, Larsen CP: Hemolysisassociated hemoglobin cast nephropathy results from a range of clinicopathologic disorders. Kidney Int 2019, 96(6):1400-1407.

19. Petejova N, Martinek A: Acute kidney injury due to rhabdomyolysis and renal replacement therapy: a critical review. \#N/A 2014, 18(3):224.

20. Group KDIGOKCW: KDIGO 2012 clinical practice guideline for the evaluation and management of chronic kidney disease. Kidney Int Supp/2013, 3(1):1-150.

21. Yamamoto G, Taura K, Ikai I, Fujikawa T, Nishitai R, Kaihara S, Zaima M, Terajima H, Yoshimura T, Koyama $Y$ et al: ALPlat criterion for the resection of hepatocellular carcinoma based on a predictive model of posthepatectomy liver failure. Surgery 2020, 167(2):410-416.

22. Ambarsari CG, Sindih RM, Saraswati M, Trihono PP: Delayed Admission and Management of Pediatric Acute Kidney Injury and Multiple Organ Dysfunction Syndrome in Children with Multiple Wasp Stings: A Case Series. Case Rep Nephrol Dial 2019, 9(3):137-148.

23. Dhanapriya J, Dineshkumar T, Sakthirajan R, Shankar P, Gopalakrishnan N, Balasubramaniyan T: Wasp sting-induced acute kidney injury. Clin Kidney J 2016, 9(2):201-204.

24. Ye TT, Gou R, Mao YN, Shen JM, He D, Deng YY: Evaluation on treatment of sustained low-efficiency hemodialysis against patients with multiple organ dysfunction syndrome following wasp stings. BMC Nephrol 2019, 20(1):240.

25. Yuan H, Lu L, Gao Z, Hu F: Risk factors of acute kidney injury induced by multiple wasp stings. Toxicon 2020, 182:1-6.

26. Xie C, Xu S, Ding F, Xie M, Lv J, Yao J, Pan D, Sun Q, Liu C, Chen T et al: Clinical features of severe wasp sting patients with dominantly toxic reaction: Analysis of 1091 cases. PLoS One 2013, 8(12).

27. Wang S, Yang L, Ci B, Maclean M, Gerber DE, Xiao G, Xie Y: Development and Validation of a Nomogram Prognostic Model for SCLC Patients. J Thorac Oncol 2018, 13(9):1338-1348.

28. Fang C, Wang W, Feng X, Sun J, Zhang Y, Zeng Y, Wang J, Chen H, Cai M, Lin J et al: Nomogram individually predicts the overall survival of patients with gastroenteropancreatic neuroendocrine neoplasms. Br J Cancer 2017, 117(10):1544-1550.

29. Huang YQ, Liang CH, He L, Tian J, Liang CS, Chen X, Ma ZL, Liu ZY: Development and Validation of a Radiomics Nomogram for Preoperative Prediction of Lymph Node Metastasis in Colorectal Cancer. $J$ Clin Oncol 2016, 34(18):2157-2164.

30. Chen X, Chen X, Wang Y, Wang X, Wang M, Liang Y, Zhu G, Jin T: A nomogram for predicting the renal dysfunction in a Chinese population with reduction in cadmium exposure based on an 8 years follow up study. Ecotoxicol Environ Saf 2020, 191:110251. 
31. Shariat SF, Capitanio U, Jeldres C, Karakiewicz PI: Can nomograms be superior to other prediction tools? BJU Int 2009, 103(4):492-495; discussion 495-497.

32. Grisotto LS, Mendes GE, Castro I, Baptista MA, Alves VA, Yu L, Burdmann EA: Mechanisms of bee venom-induced acute renal failure. Toxicon 2006, 48(1):44-54.

33. Naito A, Matsumori N, Ramamoorthy A: Dynamic membrane interactions of antibacterial and antifungal biomolecules, and amyloid peptides, revealed by solid-state NMR spectroscopy. Biochim Biophys Acta Gen Subj 2018, 1862(2):307-323.

34. Zager RA: Rhabdomyolysis and myohemoglobinuric acute renal failure. Kidney Int 1996, 49(2):314326.

\section{Figures}




\section{Patients with wasp stings $(\mathrm{n}=547)$}

Patients were excluded for missing necessary dada $(n=39)$

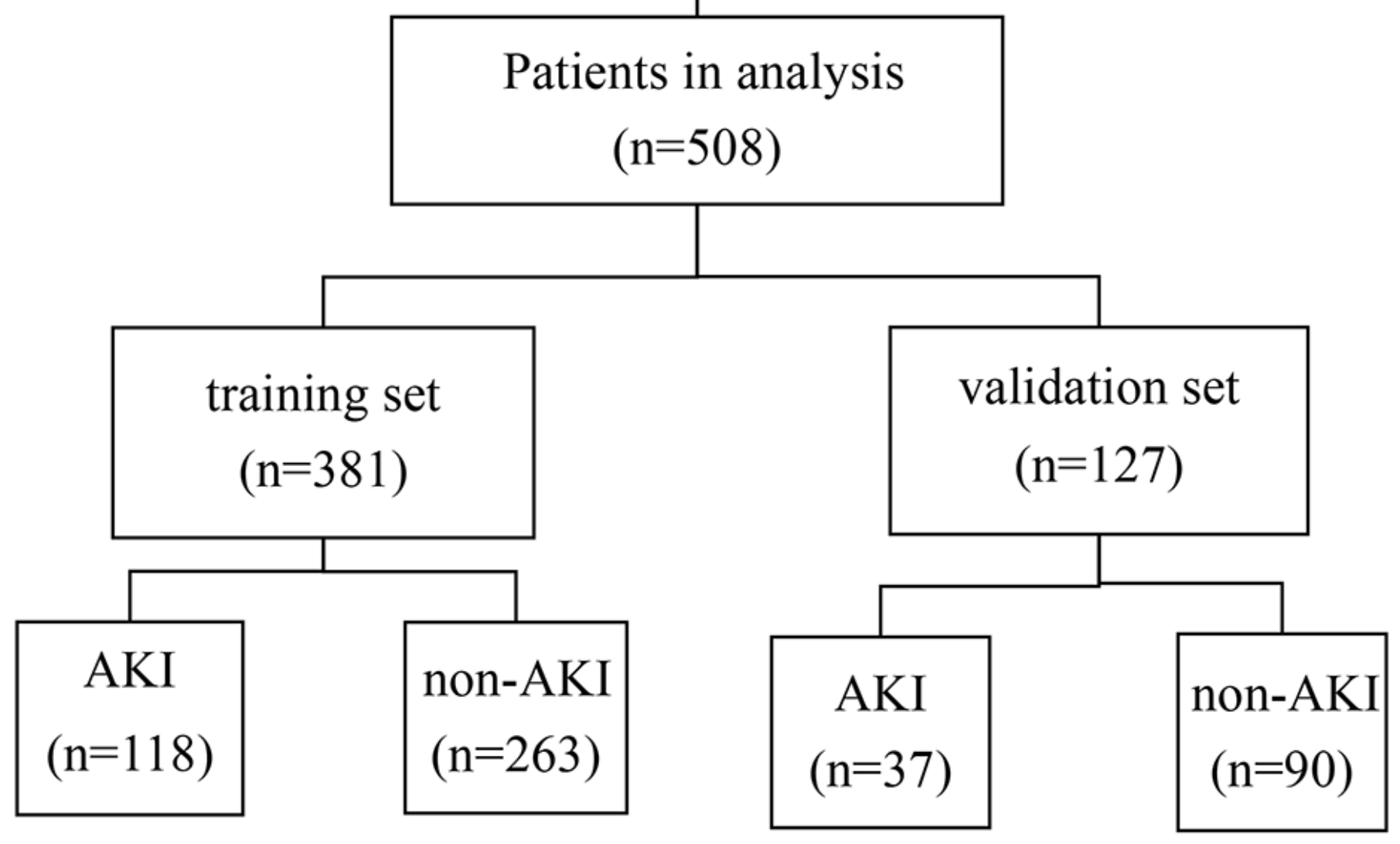

Figure 1

Flow chart of data selection 


\section{Patients with wasp stings $(\mathrm{n}=547)$}

Patients were excluded for missing necessary dada $(n=39)$

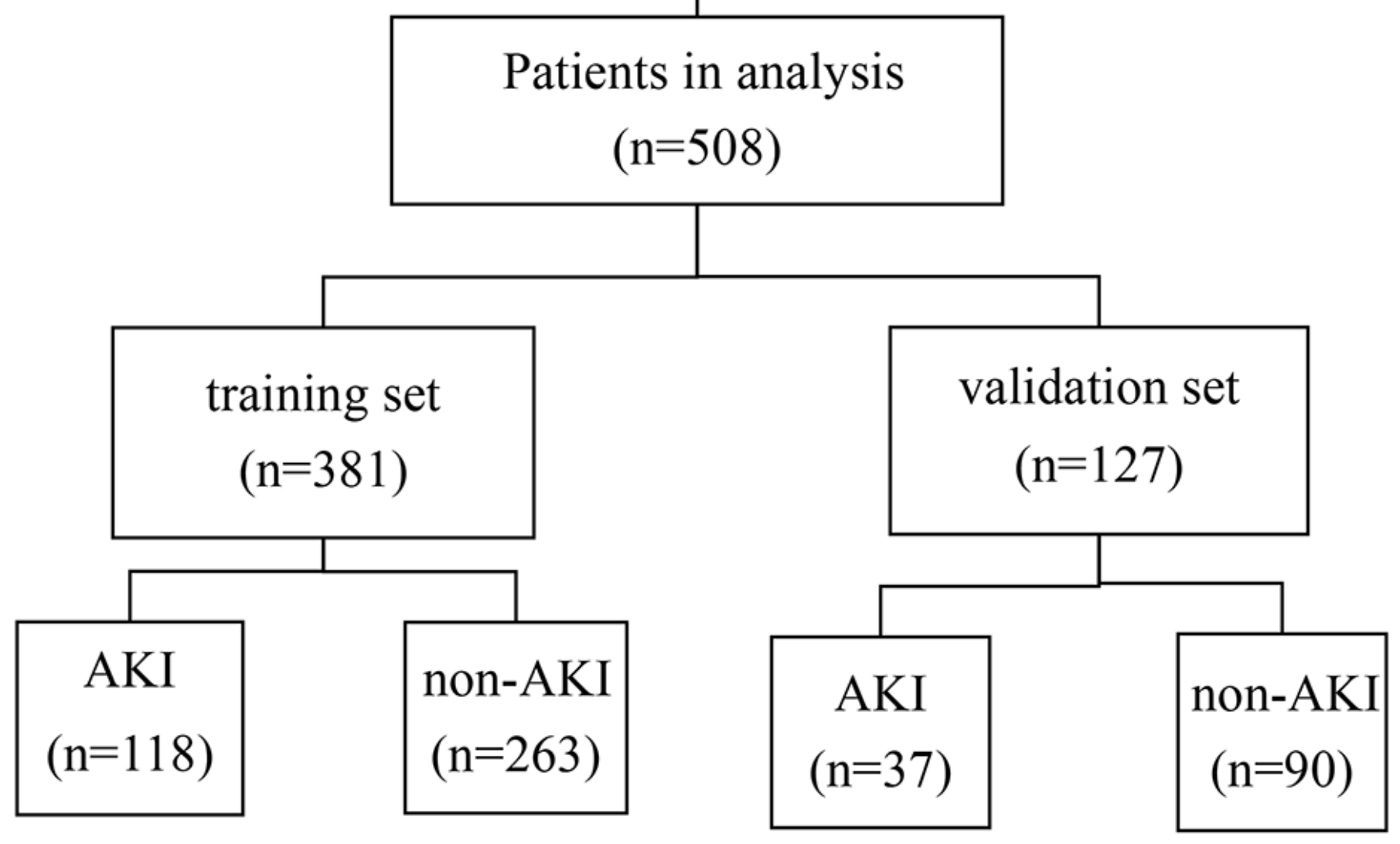

Figure 1

Flow chart of data selection 


\section{Patients with wasp stings $(\mathrm{n}=547)$}

Patients were excluded for missing necessary dada $(n=39)$

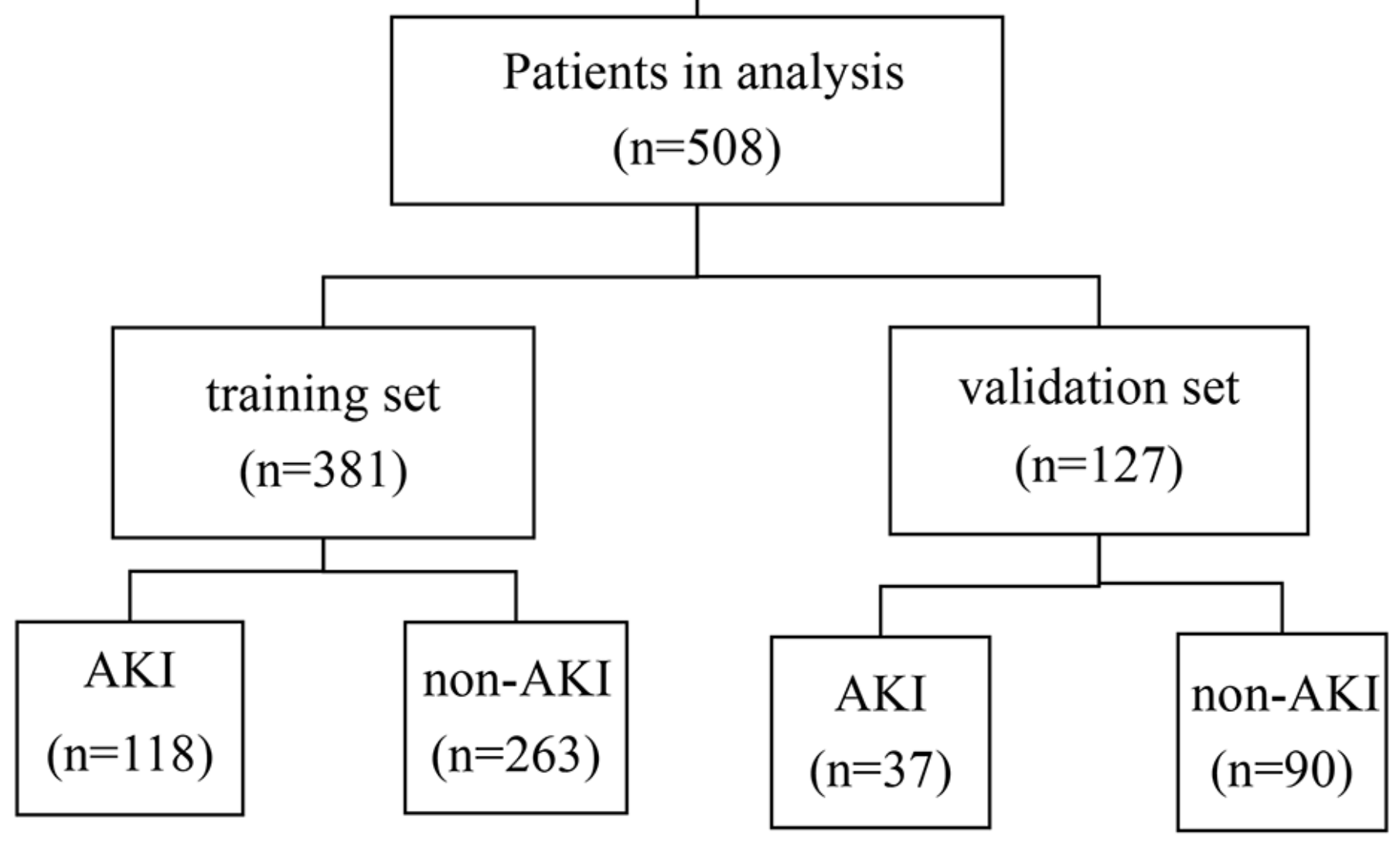

Figure 1

Flow chart of data selection 


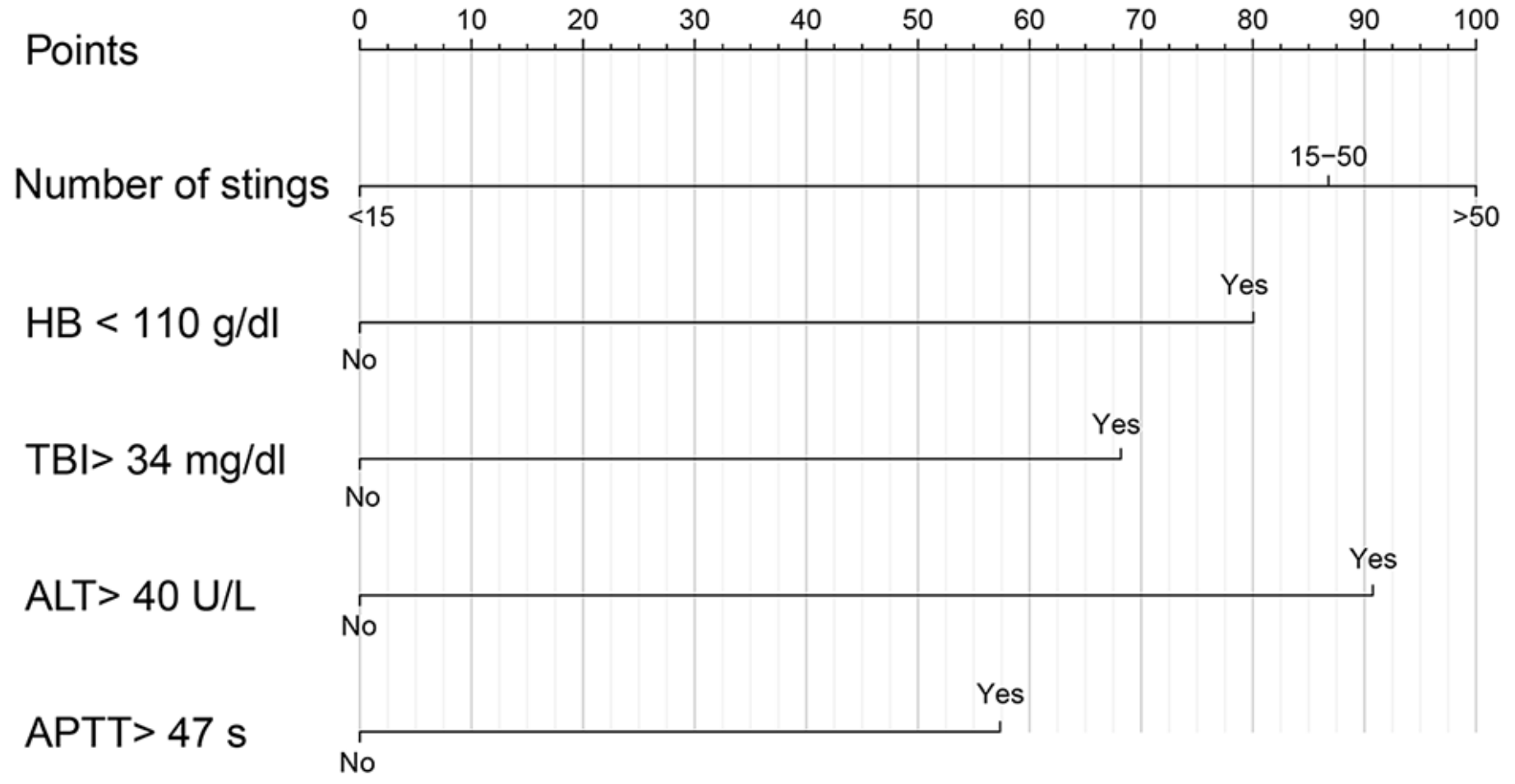

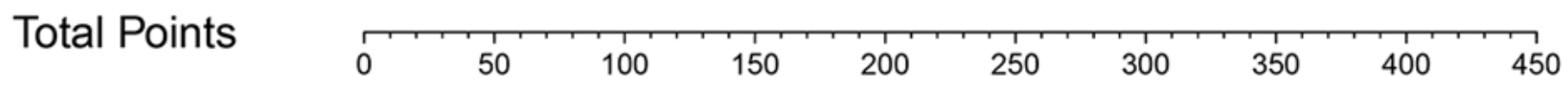

probability of AKI

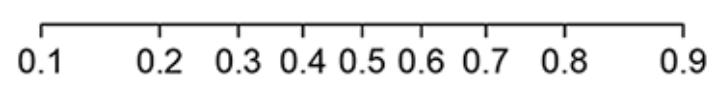

Figure 2

Nomogram for predicting AKI following wasp stings 


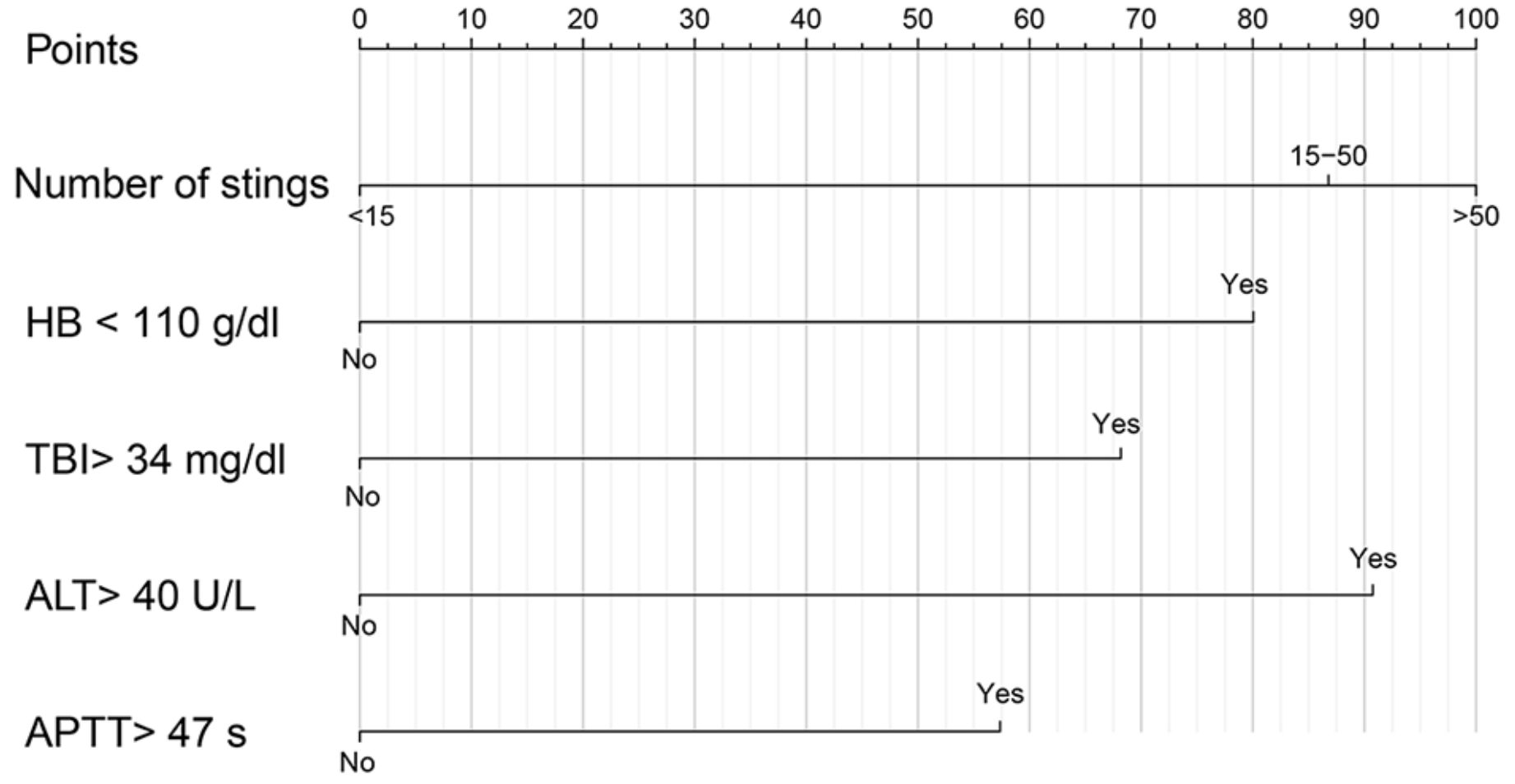

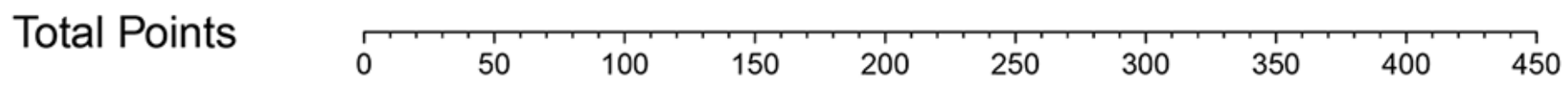

probability of AKI

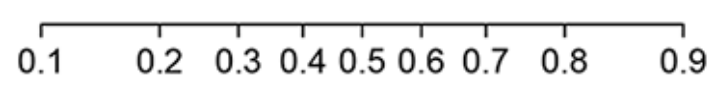

Figure 2

Nomogram for predicting AKI following wasp stings 
Points

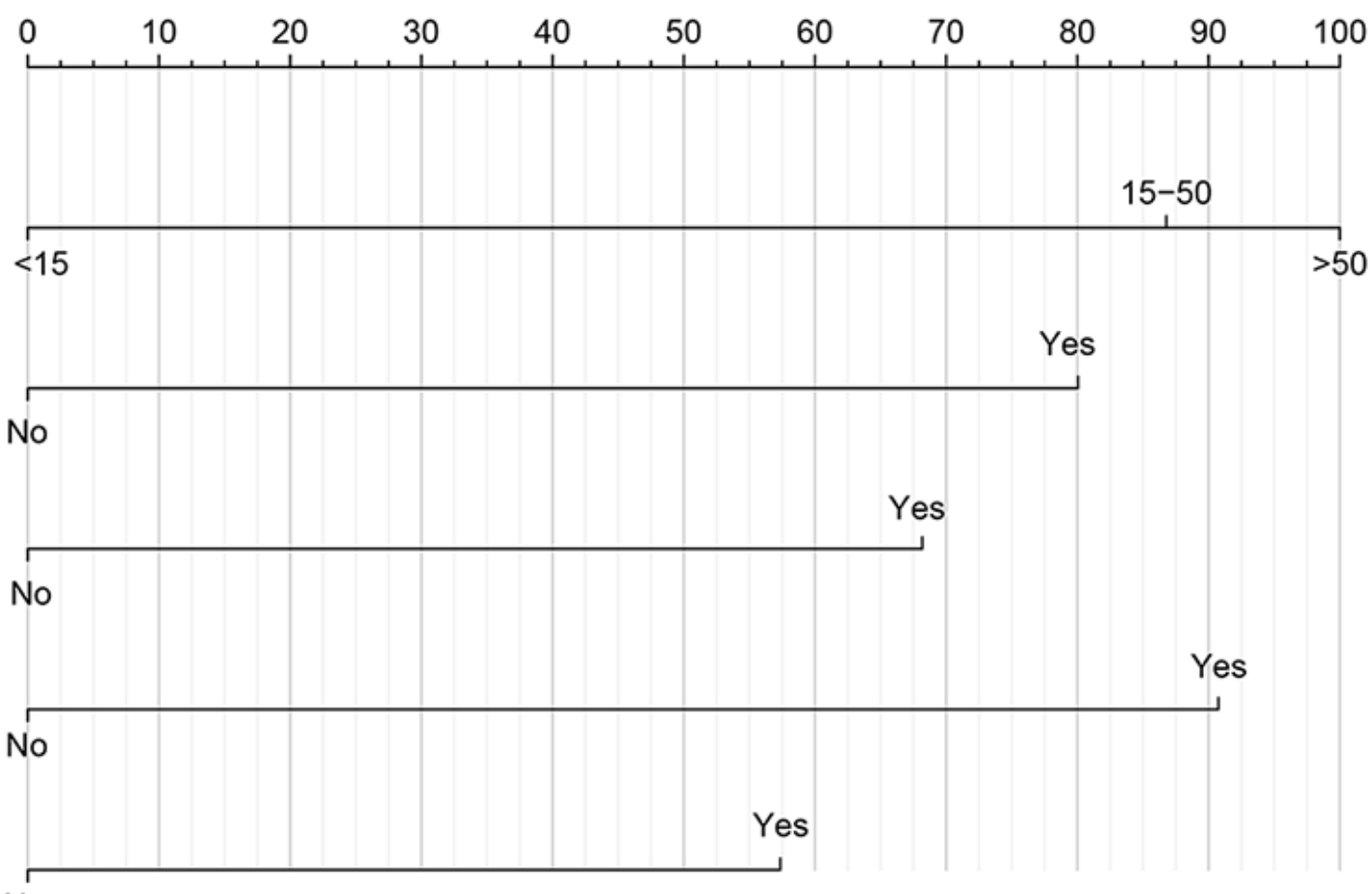

APTT $>47 \mathrm{~s}$

No

Total Points

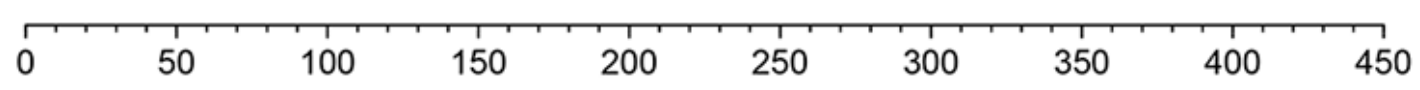

probability of AKI

$\begin{array}{llllllllll}0.1 & 0.2 & 0.3 & 0.4 & 0.5 & 0.6 & 0.7 & 0.8 & 0.9\end{array}$

Figure 2

Nomogram for predicting AKI following wasp stings
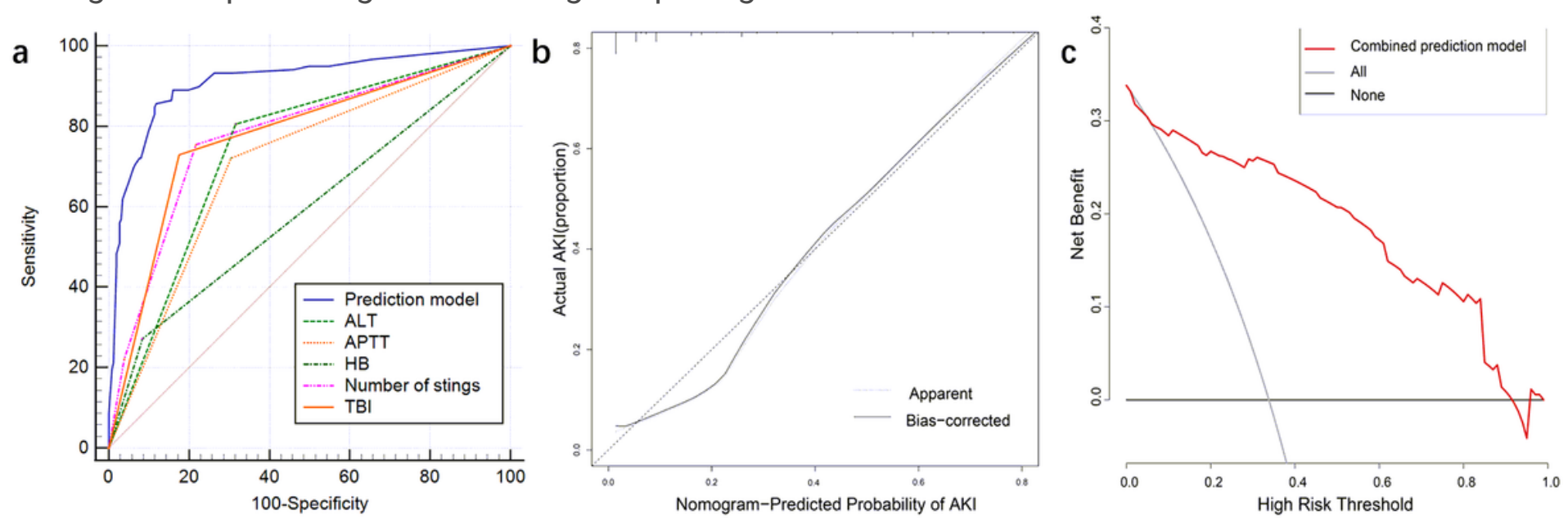

Figure 3 
Receiver operating characteristic curve, calibration curve and decision curve analysis of the model in the training set a. The receiver operating characteristic curve analysis for evaluating the accuracy of the model. b. The calibration curve depicting the correlation between nomogram-predicted probability and actual disease as internal validation. c. The decision curve analysis demonstrating the net benefit associated with the use of the nomogram-derived probability, based on multivariable logistic regression analysis, for the prediction of AKI.
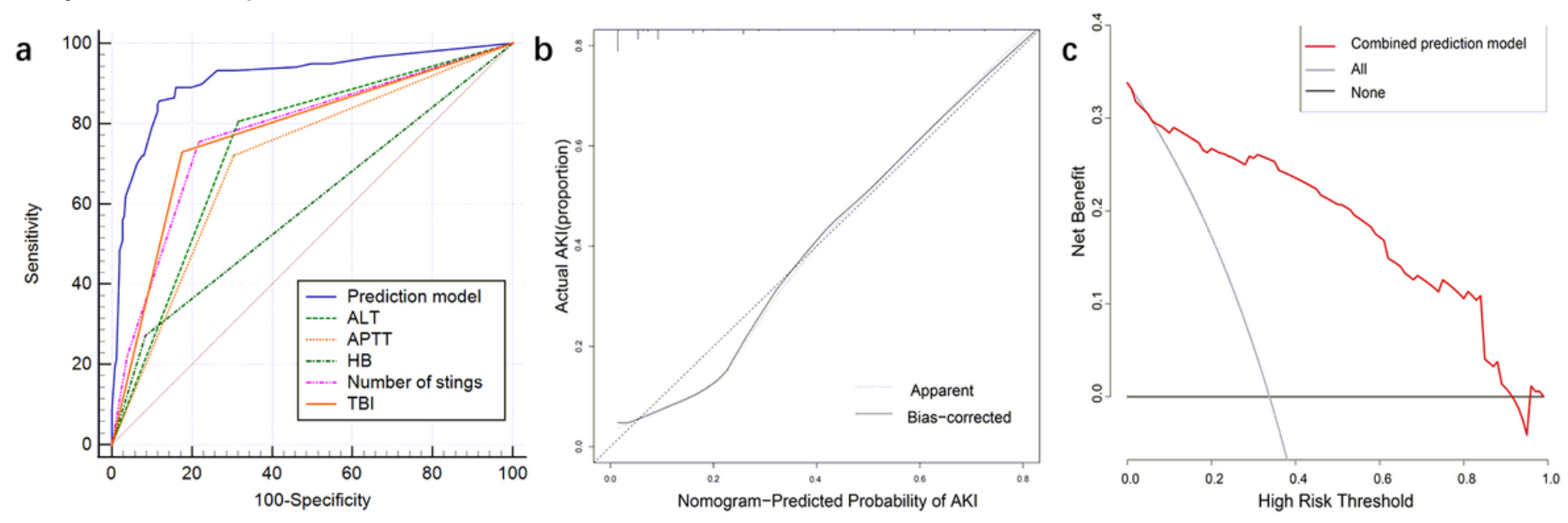

\section{Figure 3}

Receiver operating characteristic curve, calibration curve and decision curve analysis of the model in the training set a. The receiver operating characteristic curve analysis for evaluating the accuracy of the model. b. The calibration curve depicting the correlation between nomogram-predicted probability and actual disease as internal validation. c. The decision curve analysis demonstrating the net benefit associated with the use of the nomogram-derived probability, based on multivariable logistic regression analysis, for the prediction of AKI.
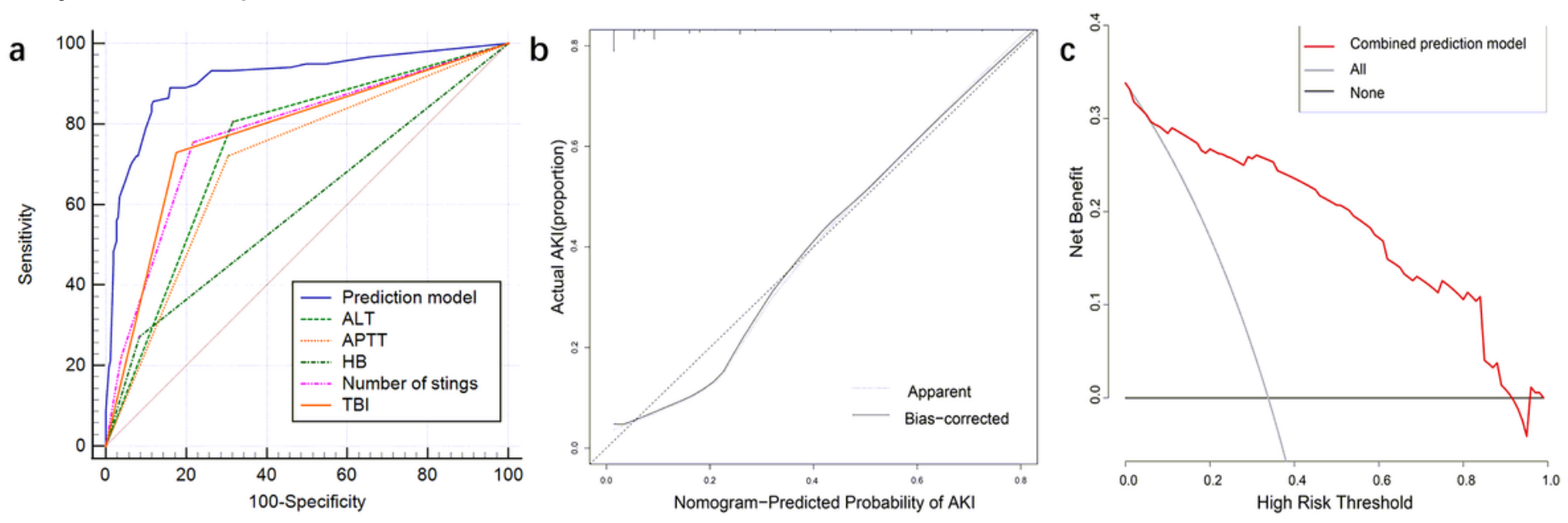

\section{Figure 3}

Receiver operating characteristic curve, calibration curve and decision curve analysis of the model in the training set a. The receiver operating characteristic curve analysis for evaluating the accuracy of the 
model. b. The calibration curve depicting the correlation between nomogram-predicted probability and actual disease as internal validation. c. The decision curve analysis demonstrating the net benefit associated with the use of the nomogram-derived probability, based on multivariable logistic regression analysis, for the prediction of AKI.

\section{Supplementary Files}

This is a list of supplementary files associated with this preprint. Click to download.

- Additionalfile1.docx

- Additionalfile1.docx

- Additionalfile1.docx

- Additionalfile2.docx

- Additionalfile2.docx

- Additionalfile2.docx

- Additionalfile3.tif

- Additionalfile3.tif

- Additionalfile3.tif

- Additionalfile4.docx

- Additionalfile4.docx

- Additionalfile4.docx

- Additionalfile5.tif

- Additionalfile5.tif

- Additionalfile5.tif

- Additionalfile6.tif

- Additionalfile6.tif

- Additionalfile6.tif

- Additionalfile7.tif

- Additionalfile7.tif

- Additionalfile7.tif 